\title{
Feature Preserving Mesh Generation from 3D Point Clouds
}

\author{
Nader Salman $^{1} \quad$ Mariette Yvinec $^{1} \quad$ Quentin Merigot $^{1}$ \\ ${ }^{1}$ INRIA Sophia-Antipolis
}

\begin{abstract}
We address the problem of generating quality surface triangle meshes from 3D point clouds sampled on piecewise smooth surfaces. Using a feature detection process based on the covariance matrices of Voronoi cells, we first extract from the point cloud a set of sharp features. Our algorithm also runs on the input point cloud a reconstruction process, such as Poisson reconstruction, providing an implicit surface. A feature preserving variant of a Delaunay refinement process is then used to generate a mesh approximating the implicit surface and containing a faithful representation of the extracted sharp edges. Such a mesh provides an enhanced trade-off between accuracy and mesh complexity. The whole process is robust to noise and made versatile through a small set of parameters which govern the mesh sizing, approximation error and shape of the elements. We demonstrate the effectiveness of our method on a variety of models including laser scanned datasets ranging from indoor to outdoor scenes.
\end{abstract}

Categories and Subject Descriptors (according to ACM CCS): [Computational Geometry and Object Modeling] [I.3.5]: Curve, surface, solid, and object representations-

\section{Introduction}

Surface reconstruction consists in inferring the shape of an object from a 3D point cloud sampled on the surface. The problem of automatic surface reconstruction from point samples has received considerable amount of efforts in the past twenty years. Surface reconstruction problems occurs in different flavors according to the quality of the data which are more or less entangled with noise, and the properties of the reconstructed object whose surface can be smooth or structured with sharp features. Moreover, an implicit description of the reconstructed object is enough for some applications, whereas some other require a data structure more amenable to further processing, like a mesh

In this paper, we focus on the case where the sampled surface is piecewise smooth and the output sought after is a high quality triangle mesh approximating the surface. Our goal is to generate from the input point cloud a mesh including a faithful representation of sharp edges, thus improving the trade-off between the accuracy and the size off the mesh. We propose for that an algorithm which experimentally proves to be fairly robust to noise.

\subsection{Related work}

Reconstruction and quality surface mesh generation are often tackled separately in the literature. We first review the approaches dealing with the problem of robust reconstruction of piecewise smooth surfaces, before reviewing the ap- proaches that combine reconstruction and quality surface mesh generation.

\subsubsection{Towards piecewise smooth reconstructions.}

In the quest to achieve both noise robustness and recovery of sharp features, we can identify an evolution of approaches ranging from smooth to sharp.

Smooth surfaces. As noise and sparseness are common in laser scanner datasets, we have seen in recent years a variety of approaches for robust reconstruction of smooth surfaces. A popular approach consists of searching an implicit function whose zero level set fits the input data points. The searched implicit functions can take different forms, being for instance approximated signed distance functions to the inferred surface [OBA05, GG07] or approximated indicator functions of the inferred solid [KBH06]. Implicit functions methods often provide spectacular robustness to noise. However most of them assume that the data points come with oriented normals and are sampled from a smooth surface. The latter assumption leads to erroneous surface reconstruction at locations where the smoothness assumption is not met.

An intermediate step toward handling sharp features consists of using locally adapted anisotropic basis functions when computing the implicit function [DTS01]. Adamson and Alexa [AA06] perform piecewise polynomial approximation through anisotropic moving least-squares (MLS). More recently, Oztireli et al. [OGG] used a kernel regres- 

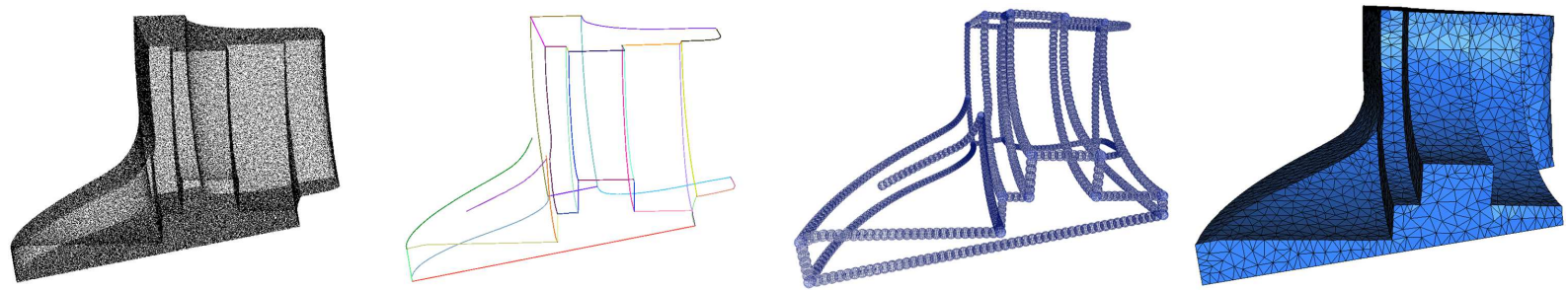

Figure 1: Overview of the method at a glance. From left to right: given a point cloud sampled on a piecewise smooth surface; detect the potential sharp edges points, cluster them with respect to the underlying sharp features direction, and extract explicit polylines for each cluster; sample protecting balls on the extracted polylines; combine extracted polylines to implicit surface reconstruction through feature preserving mesh generation to output a 3D mesh where the sharp edges are explicitly tesselated and appear as a subset of the triangle edges.

sion technique to extend the MLS reconstruction to surfaces with sharp features.

While satisfactory for some applications (e.g., visualization as opposed to mesh generation), these approaches generate surfaces which are more accurate around sharp features but are still smooth. In addition, boosting the anisotropy of a function is a dangerous game when simultaneously targeting robustness to noise, as such a strategy may amplify noise instead of the true features (features and noise are ambiguous as both are high frequency). Furthermore, a purely local distinction between smooth parts and sharp edges is likely to yield fragmented edges while a global feature extraction approach can favor long edges in order to recover the true structure.

Handling sharp features. This brings out another thread of work aimed at extracting long sharp features. Pauly et al. [PKG03] use a multi-scale approach to decide if a point is on a feature, and construct a minimum-spanning tree to recover the feature graph. Daniels et al. [DHOS07] extract featurecurves using a method based on a robust MLS projection operator which locally projects points onto the sharp edges, and grow a set of polylines through the projected points. Jenke et al. [JWSA08] extract the feature lines by robustly fitting local surface patches and by computing the intersection of close patches with dissimilar normals. Both methods show satisfactory results even for defect-laden point sets. More recently, Merigot et al [MOG09] proposed a robust estimation of curvature tensors based on a covariance measure defined from the data points Voronoi cells. Their method provides theoretical guarantees on robustness under bad sampling and noise conditions.

One way to make a local, binary distinction between smooth parts and sharp features consists of performing a local clustering of the inferred normals to the surface [OBA* 03 ]. If this process reveals more than one cluster, the algorithm does not fit just one low-degree implicit function, but as many quadrics as the number of clusters. This leads to faithful local reconstruction of a sharp edge as the intersection of two implicit primitives in the case of two clusters. A corner is reconstructed as the intersection of three or more primitives.
Note in passing that the method misses tips (sharp point that are not incident to any sharp crease), cusps and darts.

In order to achieve improved robustness, Fleishman et al. [FCOS05] segment neighborhoods of points by growing regions belonging to the same part of the surface. Robustness is obtained by using a forward search technique which finds reference planes corresponding to each region. While constituting an important progress, the method requires very dense point clouds, and is rather expensive. Also, potential instabilities in the classification can create fragmented surface parts. Targeting even higher robustness, Lipman et al. [LCOL07] enrich the MLS projection framework with sharp edges by defining a singularity indicator field based on the error of the MLS approximation. However, they choose to restrict their approach to a single singularity within each influence radius, which significantly limits the type of feature points that can be handled.

\subsubsection{Coupling reconstruction and mesh generation.}

One popular approach that couples reconstruction and mesh generation is the extended marching cubes introduced by Kobbelt et al. [KBSS01]. The input is assumed to be a discretized signed distance function enriched with oriented normals, the latter being used to decide whether or not a voxel contains a sharp feature. If a voxel is classified as containing a feature, some additional vertices are constructed within the voxel and placed at intersections between the planes defined by the vertices and their affiliated normals. This approach works robustly and efficiently by truly coupling feature extraction and mesh generation. Nevertheless, it relies on the marching cubes process which is well known to produce rather poor quality surface meshes.

Two approaches which are able to generate quality surfaces while preserving the features [DHOS07, JWSA08] proceed sequentially by first extracting a set of features, then using the advancing front method proposed by Schreiner et al. [SSFS06]. The latter produces quality isotropic meshes from different inputs including MLS representations, and can control the density of the mesh by pre-computing a guidance field. The algorithm uses time-consuming pre-processing of the point cloud in order to construct a guidance field, as well as a final post-processing using MLS projections. 
A relevant alternative to the marching cubes and advancing front strategies is the Delaunay refinement paradigm [BO05]. In this method the surface mesh is intersection free by construction as filtered out of a 3D Delaunay triangulation. A number of additional guarantees are also provided after termination of the refinement process, such as a good shape of elements, a faithful approximation of geometry and normals, and a low complexity of the mesh. More interestingly in our context, Delaunay refinement is able to couple reconstruction and mesh generation. At the intuitive level, the refinement procedure is combined with a sensing algorithm probing an implicit surface defined from the data points. The probing is performed along Voronoi edges of sampling points which are not only longer than the short edges of the marching cubes but also data-dependent as they become more and more orthogonal to the sensed surface as the refinement process goes along.

However, in [BO05] sharp features are not explicitly handled, hence they are not accurately represented in the output mesh. One way to circumvent this drawback is to extract the feature lines and to constrain the Delaunay refinement to preserve them. However, it is well known that the presence of constrained edges where meeting surface patches form small angles, endangers the termination of the Delaunay refinement [She00]. Recently Cheng et al. [CDR07, CDL07] propose to deal with this problem using the method of protecting balls set up around sharp features.

\subsection{Contributions}

In this paper, we argue that some modifications of the implicit smooth surface reconstruction algorithms can produce results with accuracy on par with the best current piecewise smooth reconstruction methods. Given a 3D point cloud sampled on a piecewise smooth surface, our algorithm consists of two steps. In the first step, we extract from the input $3 \mathrm{D}$ point cloud a set of polylines approximating the sharp edges of the object. This feature extraction is based on the robust feature estimation framework of Merigot et al. [MOG09]. In parallel, our algorithm runs on the data points a reconstruction process, providing an implicit surface, i.e. an implicit function whose zero level sets approximates the data points. The second step of our algorithm generates a mesh approximating the implicit surface while including a faithful representation of the extracted sharp edges. This step runs a feature preserving version of the Delaunay refinement meshing algorithm, based on the meshing algorithm of Boissonnat and Oudot [BO05] and using the mechanism of protecting balls introduced by Cheng et al. [CDR07, CDL07]. An overview of the method is illustrated in Figure 1.

The technical tools used in this algorithm are directly inspired by recent work [MOG09, CDL07]. However, they have not been used previously in this combination, and we argue that it is this particular synthesis of existing ideas which is the key of its success.

The main benefits of our algorithm are: Accuracy. The method provides a meshed representation of a point sampled object with an enhanced trade-off between accuracy and mesh size. Robustness. The method is robust to noise and stable under sparse sampling. Flexibility. The method offers considerable flexibility as it can be tuned with respect to the scale and the targeted size of the mesh.

\section{Feature extraction}

\subsection{Feature detection}

A point on a piecewise-smooth surface, is called a (sharp) feature point, if the surface has no tangent plane at this point. The locus of feature points on a piecewise-smooth surface has the structure of a graph.

We are given a point cloud $\mathcal{P}$ sampling a piecewise-smooth surface $S$. In order to allow the mesh generation algorithm to faithfully reproduce the sharp features of $S$, we need to extract from the input point cloud an approximation of the feature graph of $S$. Our first goal is to identify the data points that are close to sharp edges. To this aim, we use the method of Merigot et al. [MOG09] based on an analysis of the shape of Voronoi cells through their covariance matrices.

Recall that the covariance matrix of a bounded domain $E$ of $\mathbb{R}^{3}$ with respect to a base point $p$ is defined by $\operatorname{cov}(E, p)=$ $\int_{E}(x-p)(x-p)^{T} \mathrm{dx}$. The eigenvectors of this matrix capture the principal axes of $E$ while the ratio of the eigenvalues gives information on the anisotropy of $E$.

The Voronoi diagram of the sampling $\mathcal{P}$ is the cellular decomposition of the space, where each sample $p \in \mathcal{P}$ has a cell, $V(p)$, that is the locus of points closer to $p$ than to any other sample in $\mathcal{P}$ :

$$
V(p)=\left\{x \in \mathbb{R}^{d}: \forall q \in \mathcal{P},\|x-p\| \leq\|x-q\|\right\}
$$

By construction, the shape of Voronoi cells depends on the whole set of data, and some cells are unbounded. In order to get more local information, [MOG09] introduce an offset parameter $R$ and consider for each data point $p$ of $\mathcal{P}$, the covariance matrix, $M(p, R)$, of the intersection of the Voronoi cell $V(p)$ with a ball, $B(p, R)$, of radius $R$ centered at $p$ :

$$
M(p, R)=\operatorname{cov}(V(p) \cap B(p, R), p)
$$

The principal drawbacks of Voronoi-based methods is their high sensitivity to noise. To alleviate the effect of noise, [MOG09] smoothes the information contained in the covariance matrices using convolution. The method computes for each point $p$ the convolved Voronoi covariance matrix $(\mathrm{CVCM}), M^{c}(p, R, r)$, by summing the covariance matrices of all points $q$ of $\mathcal{P}$ at a distance less than $r$ to $x$ :

$$
M^{c}(p, R, r)=\sum_{q \in B(p, r)} M(q, R)
$$

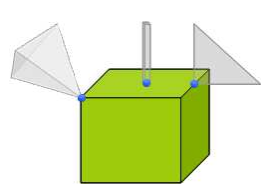

The crucial remark is that sample points lying on smooth surfaces, called smooth points, have a pencil shaped Voronoi cell; hence their CVCM has two "small" eigenvalues and a larger 
one, with the eigenvector corresponding to the large eigenvalue directed along the surface normal. Sample points that are close to sharp edges, called edge points, have rather flat Voronoi cells; therefore there CVCM has a single "small" eigenvalue and two larger ones, and the eigenvector corresponding to the small eigenvalue is directed along the edge. Sample points close to feature graph nodes, called corner points, tend to have cone-shaped Voronoi cells thus all three eigenvalues have comparable values.

Based on those observations, the algorithm we use to detect feature points (edge and corner points) is the following. We compute the CVCM, $M^{c}(p, R, r)$, of each point $p \in \mathcal{P}$, and sort the eigenvalues of this matrix by decreasing order $\lambda_{0}(p) \geq \lambda_{1}(p) \geq \lambda_{2}(p)$. If the ratio $\lambda_{0}(p) / \lambda_{1}(p)$, is greater than a threshold $t_{1}, p$ is a smooth point. Else, if the ratio between $\lambda_{1}(p) / \lambda_{2}(p)$, is greater than a threshold $t_{2}, p$ is an edge point. Else, point $p$ is a corner point. We gather, in a set $\mathcal{F}$, the pairs $\left(p, e_{2}(p)\right)$, where $p$ is a point classified as an edge point and $e_{2}(p)$ is the eigenvector of its CVCM corresponding to the smallest eigenvalue $\lambda_{2}(p)$, which is supposed to be aligned with the sharp edge direction. Note that we choose not to keep in $\mathcal{F}$, the pairs corresponding to corner points because of the instabilities of the data around these positions. Instead, we will recover corner points as extremities of sharp edges (Section 2.3).

Parameters: The method needs four parameters: the offset radius $R$, the convolution radius $r$ and both thresholds $t_{1}, t_{2}$. Parameter $R$ should be chosen smaller than to the external reach of the underlying surface defined as the smallest distance from a point of the surface to the external medial axis. Parameter $r$ has two different roles: besides smoothing the information, it determines the curvature radius under which a curved area is considered as a sharp feature. Consequently, it should be chosen depending on the noise level and on the sizing field of the targeted mesh. In all our experiments, we fixed parameter $R$ to be one tenth of the point cloud bounding sphere radius and $r$ to be a tenth of $R$, which leads to good results. Moreover, we allow the user to modify $t_{1}$ and $t_{2}$ manually, using an interactive interface.

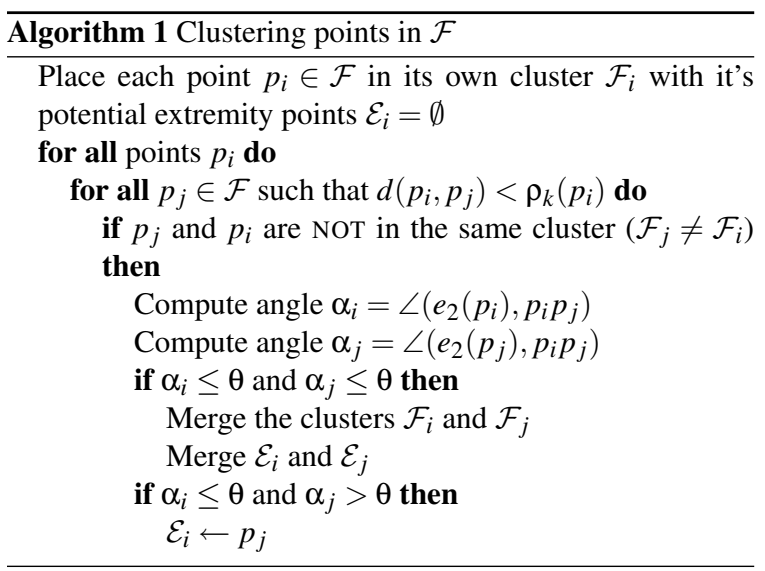

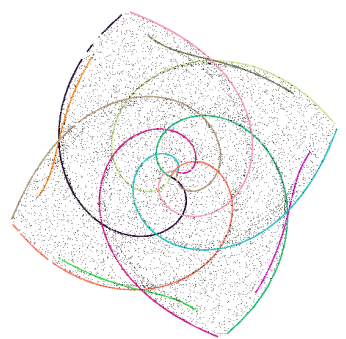

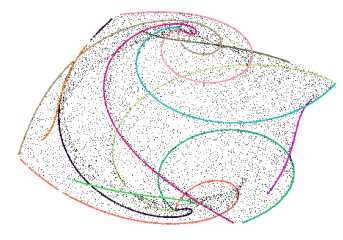

Figure 2: Result of the clustering step on the octa-flower model.

\subsection{Feature clustering}

In [MOG09], it is proven that the convolved covariance matrix is robust to noise, by deriving a bound on the quality of the results as a function of the Hausdorff distance between the point cloud and the sampled surface. However, the reconstruction of the sharp feature graph is left unaddressed in this work. Here, we need to approximate the sharp edges with polygonal lines. In their work [DHOS07] presented a technique for solving a similar problem. We however proceed differently taking advantage of the edge direction information we get from the CVCM analysis.

Our goal here is to group edge points of $\mathcal{F}$ into clusters, where each cluster samples a straight or bending sharp edge. For that, we need a similarity measure that will quantify if two edge points belong to the same sharp edge.

A pair $\left(p_{i}, p_{j}\right)$ of edge points is said to be a close pair if the Euclidean distance $d\left(p_{i}, p_{j}\right)$ is less than $\rho_{k}\left(p_{i}\right)$, where $\rho_{k}\left(p_{i}\right)=\frac{1}{k} \sqrt{\sum_{l=1}^{k}\left|p_{i}-p_{l}\right|^{2}}$, the sum running on the $k$ nearest neighbors to $p_{i}$ in $\mathcal{F}$. Two close points $p_{i}, p_{j} \in \mathcal{F}$ belong to the same cluster if the angles $\alpha_{i}=\angle\left(e_{2}\left(p_{i}\right), p_{i} p_{j}\right)$ and $\alpha_{j}=\angle\left(e_{2}\left(p_{j}\right), p_{i} p_{j}\right)$ are smaller than a given threshold $\theta$.

This criteria is injected in an union-find algorithm (Algorithm 1) that partitions points of $\mathcal{F}$ into clusters $\mathcal{F}_{i}$. In addition, if only one of the angular conditions is satisfied for a close pair $\left(p_{i}, p_{j}\right)$, say $\alpha_{i}<\theta$ but $\alpha_{j} \geq \theta$, point $p_{j}$ is classified as an extremity point of cluster $\mathcal{F}_{i}$ and included in a set $\mathcal{E}_{i}$ of extremity points related to $\mathcal{F}_{i}$. These extremity points will be of use to elongate the constructed polylines in Section 2.3, facilitating the resolution of the polylines junction recovery problem. Moreover, our implementation prunes clusters consisting of few points $\left(\left|\mathcal{F}_{i}\right| \leq 6\right)$ and unlabels the potential extremity points associated to them.

Parameters: The identification of good clusters relies on an appropriate selection of parameters $\theta$ and $k$. For big values of $\theta$ the detected clusters will be thick, thus it will be hard to sort the clustered points, a step we need for recovering the features. For our purposes, $\theta=15^{\circ}$ and $k=20$ provided sufficiently thin and representative clusters to facilitate the feature recovery procedure (Figure 2), without the need to apply the thinning heuristics of [Lee00]. 


\subsection{Feature recovery}

In the following our aim is to recover from each cluster, provided by the previous step, a polyline that will best fit the shape of the underlying sharp edge.

For each cluster $\mathcal{F}_{i}$ we build a polyline $\operatorname{Poly}\left(\mathcal{F}_{i}\right)$ by applying iteratively a procedure $\operatorname{succ}(p)$ that discovers the successor of a point $p$ (Figure 3 top). We start at a random seed point $p \in \mathcal{F}_{i}$. The successor $s(p)$ of $p$ is defined as follows: we consider the subset $N(p) \subset \mathcal{F}_{i}$ of points included in a ball of radius $r_{p}$ centered at $p$. All points in $N(p)$ are then projected onto the line defined by $p$ and the edge direction $e_{2}(p)$. Points in $N(p)$ can be divided into two subsets by inspecting the position of their projections relative to $p$. If $p$ is the starting point, we choose randomly one of the subsets to be $N^{+}(p)$. Otherwise, let $N^{+}(p)$ be the subset of points whose projections are in the direction opposite to the predecessor of $p$. Then, $s(p)$ is defined as the point in $N^{+}(p)$ with closest projection to $p$. The procedure $\operatorname{succ}(p)$ is applied iteratively until an endpoint is reached, i.e. a point for which $N^{+}(p)$ is empty. The procedure is then restarted from the seed point in the opposite direction.

The polyline $\operatorname{Poly}\left(\mathcal{F}_{i}\right)$ and the edge directions attached to its vertices are then smoothed using a standard PCA based smoothing process.

To lengthen the recovered feature polylines $\operatorname{Poly}\left(\mathcal{F}_{i}\right)$, we use the detected potential extremity points $\mathcal{E}_{i}$ attached to each cluster. For each endpoint $p \in \mathcal{F}_{i}$, if $\mathcal{E}_{i}$ is not empty, we search for neighboring points $p_{j} \in \mathcal{E}_{i}$ such as the Euclidean distance $d\left(p, p_{j}\right)<r_{p}$. All such points are projected onto the line defined by $p$ and the edge direction $e_{2}(p)$. Then, we compute the centroid $c$ of the projected points located on the half-line defined by $p$ and not containing the projection of $N(p)$. Finally, point $c$ is added to the polyline as the successor of $p$ and attached a direction $e_{2}(c)=e_{2}(p)$. Point $c$ is considered as the new endpoint of $\operatorname{Poly}\left(\mathcal{F}_{i}\right)$ (see Figure 3 bottom).

Our implementation maintains for each polyline $\operatorname{Poly}\left(\mathcal{F}_{i}\right)$ a value corresponding to the polyline length that will be of use in the feature junction recovery step to prevent joining the endpoints of a single short polyline.

Parameters: The recovered polyline result is dependent on the size of the selected neighborhood. The radius parameter $r_{p}$ should be related to the local feature size of the polyline to be recovered. Optimally, one should study the effect of the neighborhood size and propose an optimal neighborhood-size-selection scheme. Practically, we do not have this information, hence we choose $r_{p}=\rho_{k}(p)$ where $k=5$

Junction recovery. In order to build a realistic approximation of the sharp feature graph, we need to construct appropriate junctions between the constructed polylines. The task is simplified as we have extended the polylines and thus reduced the search space for potential junctions. Note that

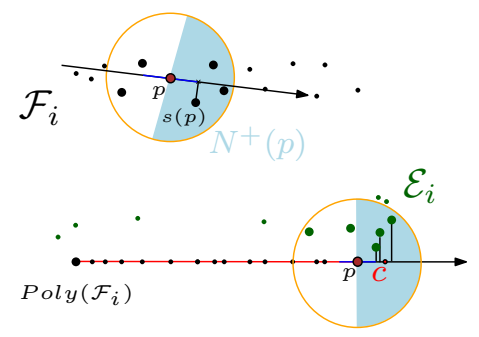

Figure 3: Illustration of feature recovery step. Top: procedure $\operatorname{succ}(p)$ that discovers the successor, $s(p)$, of a point $p \in \mathcal{F}_{i}$. Bottom: after smoothing the polyline $\operatorname{Poly}\left(\mathcal{F}_{i}\right)$, we elongate it at each endpoint $p$ using the detected extremity points $\mathcal{E}_{i}$

sharp edges joined at vertices of the feature graph are classified as follows: a dart is incident to a single sharp edge; a cusp is incident to two sharp edges forming a sharp angle at the junction; a corner is incident to three or more sharp edges.

A natural solution is to merge in a single vertex, polylines endpoints that are spatially close. We could simply take as position for the merged vertex the barycenter of the merged endpoints. However, to increase the accuracy of the approximation of sharp feature junctions we proceed as follows.

For each endpoint $p$ of $\operatorname{Poly}\left(\mathcal{F}_{i}\right)$, we start by constructing the set $\mathcal{J}(p)$ of endpoints that are within a ball $B\left(p, r_{j u n c}\right)$ of radius $r_{\text {junc }}$ centered at $p$. In case there is no endpoint in $\mathcal{J}(p)$ other than $p$ itself, we tag $p$ as a dart and continue. Otherwise, $|\mathcal{J}(p)| \geq 2$ and we compute the junction position as follows. For every pair of endpoints $p_{j}, p_{k} \in \mathcal{J}(p)$, we consider the two points $q_{j}, q_{k}$ which are the closest points such that $q_{j}$ belongs to the line $L_{j}$ passing through $p_{j}$ and di-

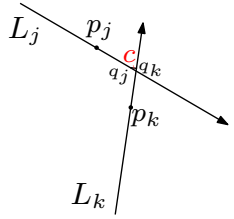
rected along $e_{2}\left(p_{j}\right)$ and $q_{k}$ belongs to the line $L_{k}$ passing through $p_{k}$ and directed along $e_{2}\left(p_{k}\right)$. The junction point is computed as the barycenter of the set of closest points, except that each closest point that is not in the ball $B\left(p, r_{j u n c}\right)$ is replaced by the endpoint $p$ in the barycenter computation.

Our procedure has the advantage of being easy to implement and is computationally inexpensive since it does not introduce any plane nor surface fitting and intersection computations. An example of finding junctions from polylines including three junction cases, darts, cusps and corners is illustrated in Figure 5. Note that we do not capture tips, i.e. vertices incident to zero sharp edges but are sharp.

Parameters: The radius parameter $r_{\text {junc }}$ should be chosen large enough, in order to cover the empty space between polyline endpoints. On the other hand, $r_{\text {junc }}$ generally shouldn't be larger than the length of the shortest polyline - which would lead to both of its endpoints being merged into a single junction. In practice, we found that choosing $r_{\text {junc }}$ to be equal to five times the average spacing of the ending four points of a polyline leads to good results. 


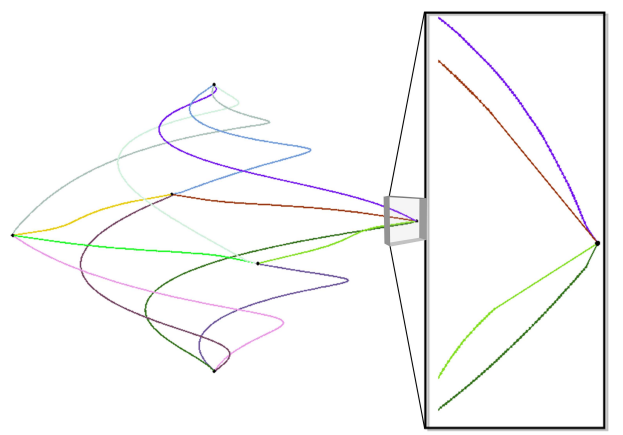

Figure 4: Left: Feature recovery for the octa-flower model. Right: Closeup view of the recovered sharp edges and the recovered junction (black dot).

\section{Feature preserving mesh generation}

Besides extracting sharp edges, our algorithm runs on the data points a reconstruction process, such as Poisson reconstruction [KBH06] or any variant of moving least square [GG07], providing an implicit description of a surface approximating the data points. Such an implicit surface is represented as a function $\left(\mathbb{R}^{3} \rightarrow \mathbb{R}\right)$ whose zero level set approximates the data points. Our goal in this section is to show how, using a feature preserving variant of a Delaunay refinement mesh generation algorithm, we obtain a surface mesh approximating the implicit surface and including a faithful representation of the sharp edges extracted in the previous section.

\subsection{Delaunay refinement surface mesh generation}

To generate a mesh approximating the implicit surface, we use the surface meshing algorithm of Boissonnat and Oudot [BO05]. This algorithm is a Delaunay refinement, based on the notion of restricted Delaunay triangulation.

Let $\mathcal{P}$ be a set of points in $\mathbb{R}^{3}$ and $\mathcal{S}$ be a surface. The restricted Delaunay triangulation, $\mathcal{D}_{\mathcal{S}}(\mathcal{P})$, is the sub-complex of the Delaunay triangulation $\mathcal{D}(\mathcal{P})$ formed by the faces of $\mathcal{D}(\mathcal{P})$ whose dual Voronoi faces intersect $\mathcal{S}$. It has been proven that if $\mathcal{P}$ is a "sufficiently dense" sample of $\mathcal{S}$, $\mathcal{D}_{\mathcal{S}}(\mathcal{P})$ is homeomorph to the surface $\mathcal{S}$ and is a good approximation of $\mathcal{S}$ in the Hausdorff sense [ES97, AB99, BO05].

The meshing algorithm of [BO05] builds on this result. It maintains the restricted Delaunay triangulation $\mathcal{D}_{\mathcal{S}}(\mathcal{P})$ of a sampling $\mathcal{P}$ of the surface, and refines the sampling $P$ until it is dense enough for the restricted Delaunay triangulation to be a good approximation of the surface. More precisely, the refinement process tracks bad facets in $\mathcal{D}_{\mathcal{S}}(\mathcal{P})$, i.e. facets that do not comply to the meshing criteria. Each bad facet is killed out by the insertion of a new vertex at its surface center which is the point where the Voronoi edge, dual to the facet, intersects the surface.

Such a Delaunay refinement surface meshing algorithm yields a quality surface mesh, free of self-intersections.

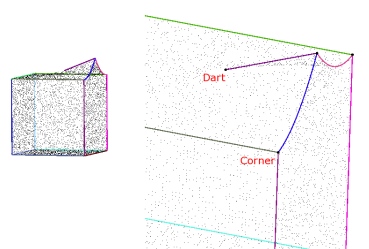

(a)

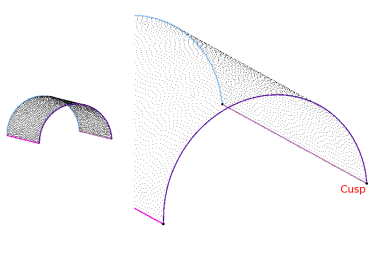

(b)
Figure 5: Junction recovery. (a) a dart and multiple corners are recovered from the smooth feature model; (b) multiple border cusps are recovered from the half cylinder model

However, since features are not handled explicitly, they are poorly represented in the output mesh (see Figure 7 middle).

Parameters: The parameters of the Delaunay refinement are the parameters of the criteria defining the bad facets. A facet is bad if either some facet angle is smaller than $\alpha$, or some facet edge is longer than $l$ or the distance between the center of the facet and the surface is more than $d$.

\subsection{Feature preserving extension}

To achieve an accurate representation of the extracted sharp features in the final mesh, we resort to the method of protecting balls proposed by Cheng et al. [CDR07] and experimented in [CDL07]. This method performs, before the Delaunay refinement, a protecting step in which each sharp feature is covered by a set of protecting balls, such that:

$\triangleright$ protecting balls are centered on sharp features and each feauture is completely covered by the union of protecting balls centered on it;

$\triangleright$ any two balls have an empty intersection, except balls with consecutive centers on a given sharp feature that intersect significatively but do not include each other's center; $\triangleright$ any three balls have an empty intersection.

Once the protecting balls have been computed, they are regarded as weighted points and inserted altogether, as initial vertices, in a weighted Delaunay triangulation. The Delaunay refinement process is then performed using this weighted Delaunay triangulation: at each step, the refinement process computes the weighted version of a bad facet surface center. This point is affected a zero weight and inserted in the weigthed Delaunay triangulation.

Such a weight setting, together with the protecting ball properties, enforces the fact that two protecting ball centers, consecutive on a sharp feature polyline are guaranteed to remain connected by a restricted Delaunay edge. Furthermore, owing to the fact that no three balls intersect, the refinement process never inserts refinement point in the union of the protecting balls nor probes the surface in this protected region. This ensures the termination of the refinement process whatever may be the dihedral angle formed by smooth patches incident on a sharp feature. 

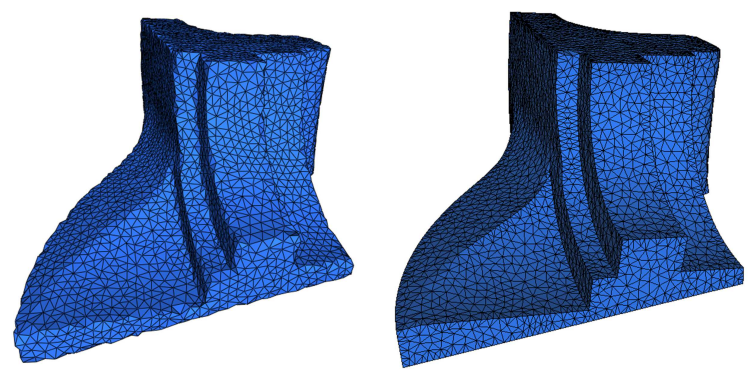

(a) $\delta=3.5$
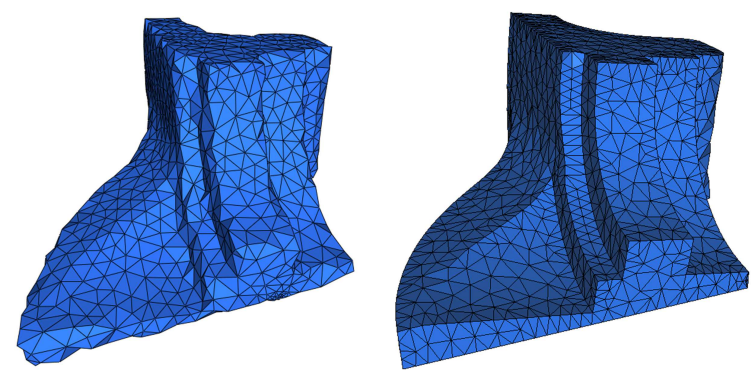

(b) $\delta=7$

Figure 6: Feature preserving mesh generation for the fandisk model. The threshold $\delta$ is used to adjust the size of the protective balls thus the mesh resolution; for $(a)$ and $(b)$, the meshed Poisson implicit function (PIF) is on the left whereas the meshes generated with our feature preserving extension using PIF are on the right. For both threshold values the extracted polylines and their endpoints are tesselated explicitly.

Protecting balls have a crucial influence on the sizing of the output mesh. On one hand protecting ball centers define the approximation of sharp features in the final mesh, and the sizing parameter $l$ of the Delau-

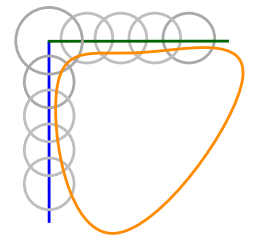
nay refinement cannot be smaller than the spacing between protecting ball centers. On the other hand, to provide a surface mesh with facets incident to the mesh edges approximating a sharp feauture, the refinement process requires that the union of protecting balls covers the gap between the implicit surface (orange) and the extracted feature polylines (green, blue).

In practice, to compute the protecting balls, we first keep all endpoint vertices of the detected polylines $\operatorname{Poly}\left(\mathcal{F}_{i}\right)$ and then sample points on $\operatorname{Poly}\left(\mathcal{F}_{i}\right)$ according to some user defined uniform distance $d^{\prime}$. Then the algorithm tries setting a uniform weight $w_{p}=2 / 3 * d^{\prime}$ on sample points. If the resulting set of balls do not comply with the above rules, it is locally fixed by adding new sample points and reducing ball radii.

Parameters: In our experiments, the mesh generation step depends on a single user-defined parameter $\delta$. Indeed, we use as unit length the quantity avg defined as the mean of the distances from each input data point to each one of its six first nearest neighbors. Then, we fix the protecting ball parameter and Delaunay refinement parameters to: $d^{\prime}=\delta * a v g, \alpha=25^{\circ}, l=2 * d^{\prime}, d=0.6 * d^{\prime}$.

\section{Experimental results}

Our prototype is implemented in $\mathrm{C}++$ using the robust primitives provided by the CGAL library [cga]. We use CGAL 3D triangulation as the core data structure to compute the Voronoi covariance matrices of the input point cloud [MOG09]. The CGAL library also provides us with an implementation of the Poisson reconstruction method [KBH06] to compute an implicit surface from the input point cloud. Lastly CGAL provides an implementation of the Delaunay refinement surface meshing algorithm of Boissonnat and Oudot [BO05].

A crucial component for reaching good timings is the efficient answer to queries for the subset of a point cloud contained in a given ball. Such a query is required for computing the CVCM used to detect edge points, it is also used for clustering edge points, and finally for recovering and joining the feature polylines. We have chosen to implement these queries using the ANN library [ann]. Note that Poisson reconstruction assumes that the input points come with oriented normals. Here, we take advantage of the result from the eigenvector analysis of the CVCM. Each data point is attached a normal direction corresponding to the eigenvector of its CVCM with largest eigenvalue. The normals are oriented using the method described by Hoppe et al. in [HDD $\left.{ }^{*} 92\right]$ and provided by the point set processing package of CGAL.

Experiments where conducted on various datasets, we report here some of them: five "clean" synthetic models (fandisk, octa-flower, carved object, blade, block), a synthetic model with added noise (fandisk), the raw output of a Minolta ${ }^{\mathrm{TM}}$ laser scanner for an indoor model (Ramses), and the raw output of a Leica ${ }^{\mathrm{TM}}$ laser scanner for an outdoor urban scene (Church of Lans le Villard).

All results presented here have been produced using fixed values of the parameters that are mentioned in the Parameters paragraph at each of the algorithm steps, except for the three parameters $t_{1}, t_{2}$ and $\delta$.

Accuracy versus mesh size. Figure 6, illustrates the effect of parameter $\delta$ on the output mesh resolution. Here we have chosen $t_{1}=50, t_{2}=6$ and protected the extracted polylines with balls obtained for respectively $\delta=3.5$ (a), $\delta=7$ for (b).

We further evaluated our method on the three synthetic datasets, shown in Figure 7. The octa-flower model is interesting as it has curvy, sharp features. The two other models carved_object, and blade show the ability of our approach to 

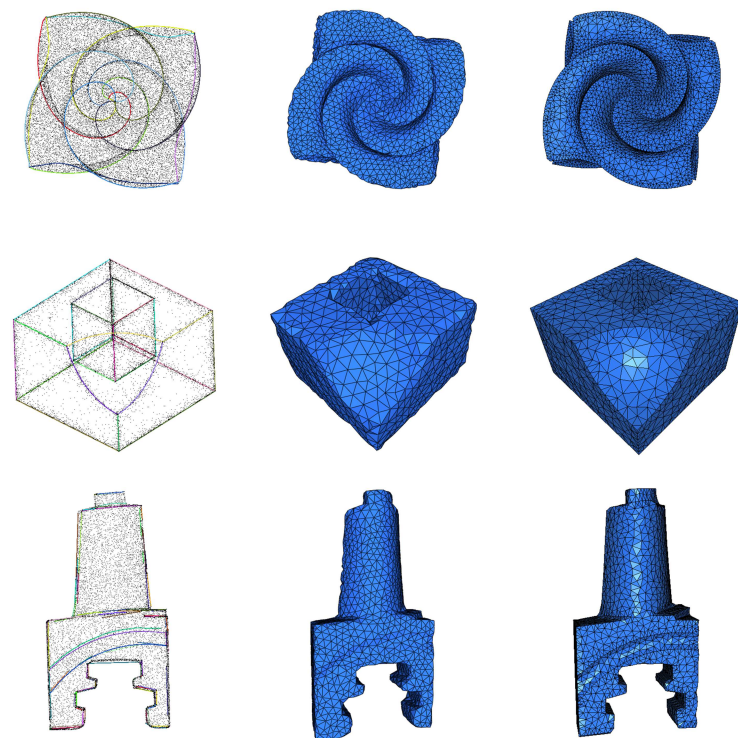

Figure 7: Feature preserving mesh generation on synthetic data sets. From left to right: Our extracted polylines overlayed to the input point cloud; Delaunay refinement mesh of the Poisson implicit surface; output of our feature preserving mesh generation. Top: octa-flower; middle: carved_object (courtesy of P. Jenke); and bottom: blade models

recover sharp edges from sparse samplings ( $\leq 50 \mathrm{k}$ points). Notice, that in the case of carved_object and blade, the original models are 3D meshes, but the vertices of those meshes are NOT lying on the feature lines. The initial triangle edges are zigzaggy along the feature lines.

As already mentioned, our goal is to improve the accuracy for a given mesh size, by ensuring a faithful representation of sharp edges. To illustrate our achievement with repect to this goal, we show, for each model in Figure 7, two meshes computed with the same value or parameter $\delta$, hence the same value of Delaunay refinement algorithm parameters $\left(\alpha=25^{\circ}, l=2 * d^{\prime}, d=0.6 * d^{\prime}\right)$

For quantitative evaluation of the accuracy improvement, Figure 8 shows an approximation of the Hausdorff distance between generated meshes and the ground truth model for various size of the generated mesh. Note that, the feature preserving Delaunay refinement (FPE) always outperforms standard Delaunay refinement (PIF) and that the effect is all the more important for coarse meshes.

Figure 9 is another illustration of the ability of our method to enhance the trade-off between accuracy and mesh size through feature extraction and preservation. Note that the only way for standard mesh generation methods (marching cubes and Delaunay" refinement) to overcome the lack of feature lines is to generate high resolution meshes.

Complex datasets. In order to evaluate the ability of our feature extraction method to cope with noise, we perturbed

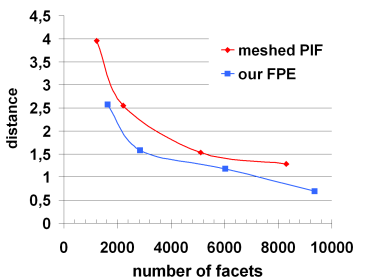

(a) fandisk

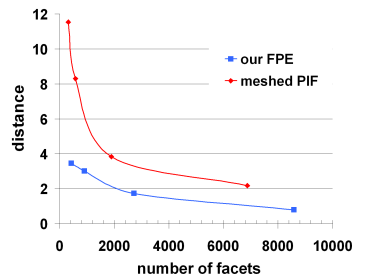

(b) block
Figure 8: Quantitative evaluation of the accuracy versus mesh size : (a) the fandisk model, points on the curve correpond to values 10, 7, 4.5 and 3.5 of $\delta(b)$ the block model, points on the curve correpond to values 9, 6, 3 and 1.5 of $\delta$. The Hausdorff distance plotted vertically is given in units of $\frac{1}{200}$ of the bounding box diagonal length.

the points sampled on the fandisk model. The perturbation is uniform and has an amplitude of $1 \%$ of the diagonal of the dataset bounding box. Keeping the same set of parameters $\left(t_{1}, t_{2}\right)$ as for the unperturbed fandisk model, Figure 10 shows the detected edge points. Although the edge points are more diffused with this amount of noise, the computed feature directions remain quite stable. Moreover, despite introducing strong noise, the feature extraction step constructed almost all sharp edges.

To evaluate the practical usability of this new approach, we applied it to two laser scan datasets acquired from two different environments. The first one is an indoor laser acquisition of the Ramses model, Figure 11. The second is an outdoor laser acquisition of the Church of Lans le Villard (France), Figure 12. These datasets pose some problems compared to the synthetic models presented previously as near sharp features data are sparse and entangled with a high level of noise.

The total time required to construct the feature polylines depends on the number of detected sharp edges points. Our prototype program has not been optimized and the details

\begin{tabular}{l|c|c|cccc|c} 
Model/ & Parameters & $|\mathcal{F}|$ & \multicolumn{5}{|c}{ Timings $[\mathrm{sec}]$} \\
\#points & $t_{1}, t_{2}, \delta$ & & $(1)$ & $(2)$ & $(3)$ & $(4)$ & Fig. \\
\hline fandisk/200k & $50,6,3.5$ & $2 \mathrm{k}$ & 153 & 17 & 20 & 24 & 6 \\
& $50,6,7$ & $2 \mathrm{k}$ & 153 & 17 & 20 & 7 & 6 \\
noise 1\% & $50,6,7$ & $3 \mathrm{k}$ & 126 & 32 & 41 & 7 & 10 \\
\hline octa-flower/167k & $25,4,3.5$ & $3 \mathrm{k}$ & 96 & 19 & 53 & 13 & 7 \\
\hline carved_object/30k & $16,4,4$ & $2 \mathrm{k}$ & 18 & 13 & 18 & 12 & 7 \\
\hline blade/50k & $100,7,2.5$ & $4 \mathrm{k}$ & 34 & 52 & 32 & 9 & 7 \\
\hline block/40k & $8,2,6$ & $2 \mathrm{k}$ & 16 & 20 & 37 & 7 & 9 \\
\hline Ramses/210k & $150,7,5$ & $8 \mathrm{k}$ & 108 & 112 & 67 & 17 & 11 \\
\hline Lans church/500k & $90,10,5$ & $17 \mathrm{k}$ & 368 & 306 & 184 & 78 & 12 \\
\hline
\end{tabular}

Table 1: Statistics of our prototype implementation. The timings are given for each of the 4 stages in our method: sharp edges points detection (1); clustering (2); polyline construction and junction (3); and feature preserving mesh generation (4). 

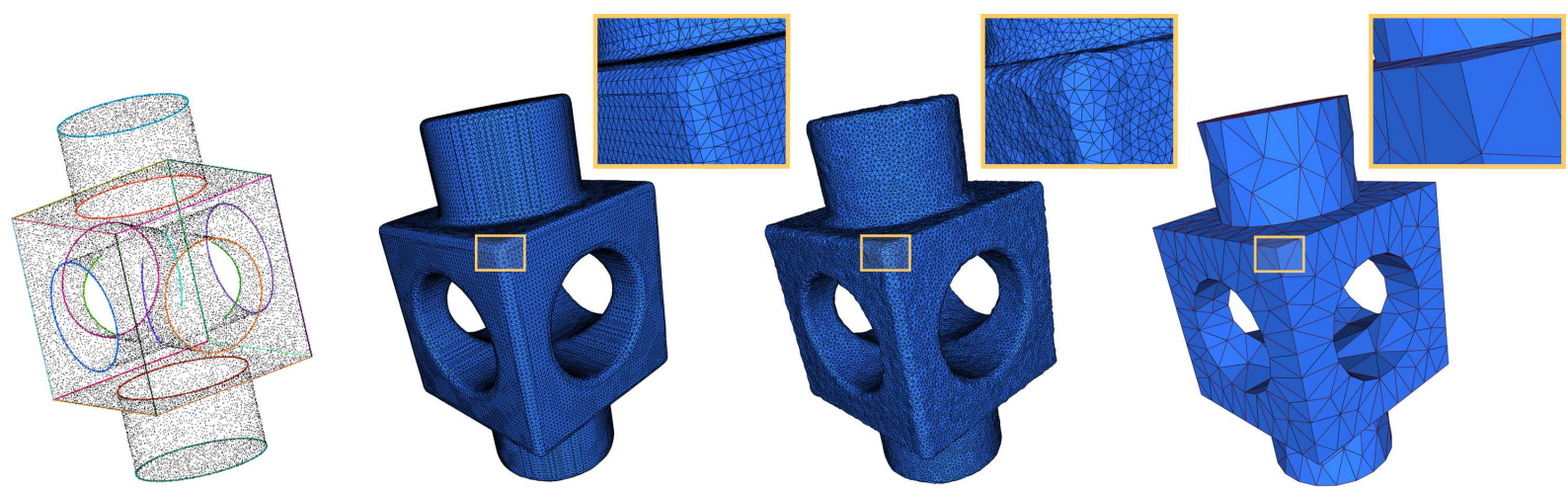

Figure 9: From left to right: input point cloud and overlayed extracted feature polylines for the block model and three generated meshes from the implicit surface obtained by Poisson reconstruction: marching cubes implementation [KBH06], with default octree depth, (62k facets); standard Delaunay refinement [BO05], with $\delta=0.5$, (60k facets); our feature preserving Delaunay refinement, with $\delta=6$, (900 facets).
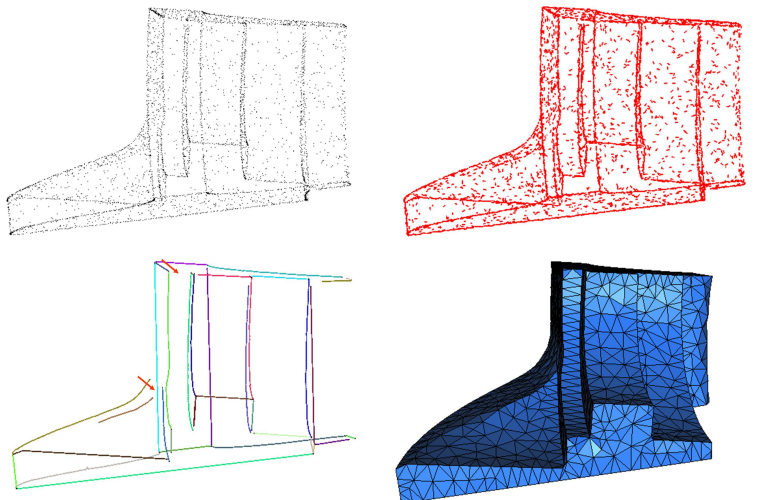

Figure 10: Feature extraction and preserving mesh generation for the fandisk model corrupted with uniform noise of $1 \%$ of its bounding box. From top to bottom, left to right: the detected edge points $\left(t_{1}=50, t_{2}=6\right)$; the associated feature directions; the extracted feature polylines; the output mesh with $(\delta=7)$. Note how choosing large protective balls patches the small gaps (pointed by small arrows) between feature polylines.

shown in Table 1 are just given for indication (on a laptop with $3.5 \mathrm{~Gb}$ memory, Intel $\AA$ Core $^{\mathrm{TM}} 22.7 \mathrm{Ghz}$ processor).

Limitations. Since feature extraction and smooth surface reconstruction are run independently, the extracted feature graph may have a certain discrepancy with respect to the smooth reconstructed surface. The feature preserving mesh generation algorithm yields a surface mesh with a faithfull representation of sharp features provided that the protecting balls cover the gap between the extracted features and the reconstructed smooth surface. This condition puts one more constraint on the parameter $\delta$ and, hence on the mesh accuracy and sizing.

The pipeline currently described does not support adaptative sampling and the generation of meshes with non uniform sizing field. This however is not a real limitation of the method
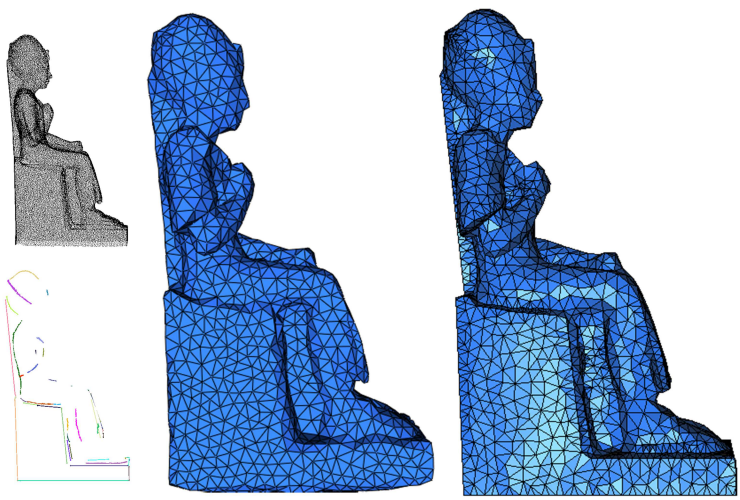

Figure 11: Left: input point cloud of Ramses model downsampled to about $27 \%$ of the original dataset (top), extracted feature polylines (bottom). Middle: meshed Poisson implicit function. Right: output of our feature preserving mesh generation ( $5 k$ facets).

but simply results from our concern to limit and simplify the parameters of the algorithm.

\section{Conclusion}

In this paper, we have presented an efficient and robust feature preserving mesh generation strategy from 3D point clouds. The approach we use builds a bridge between implicit surface reconstruction and mesh generation.

As for future work, it would be interesting to explore the possibility to automatically pre-compute the different parameters from the input point cloud. We are also planning to exploit the mesh generation algorithm flexibility to apply our method to more challenging datasets provided by multi-view stereo reconstruction. We also plan to make our code available very soon and we hope that it will be useful in the fields which require simplified and realistic geometric models, like in urban modelling. 


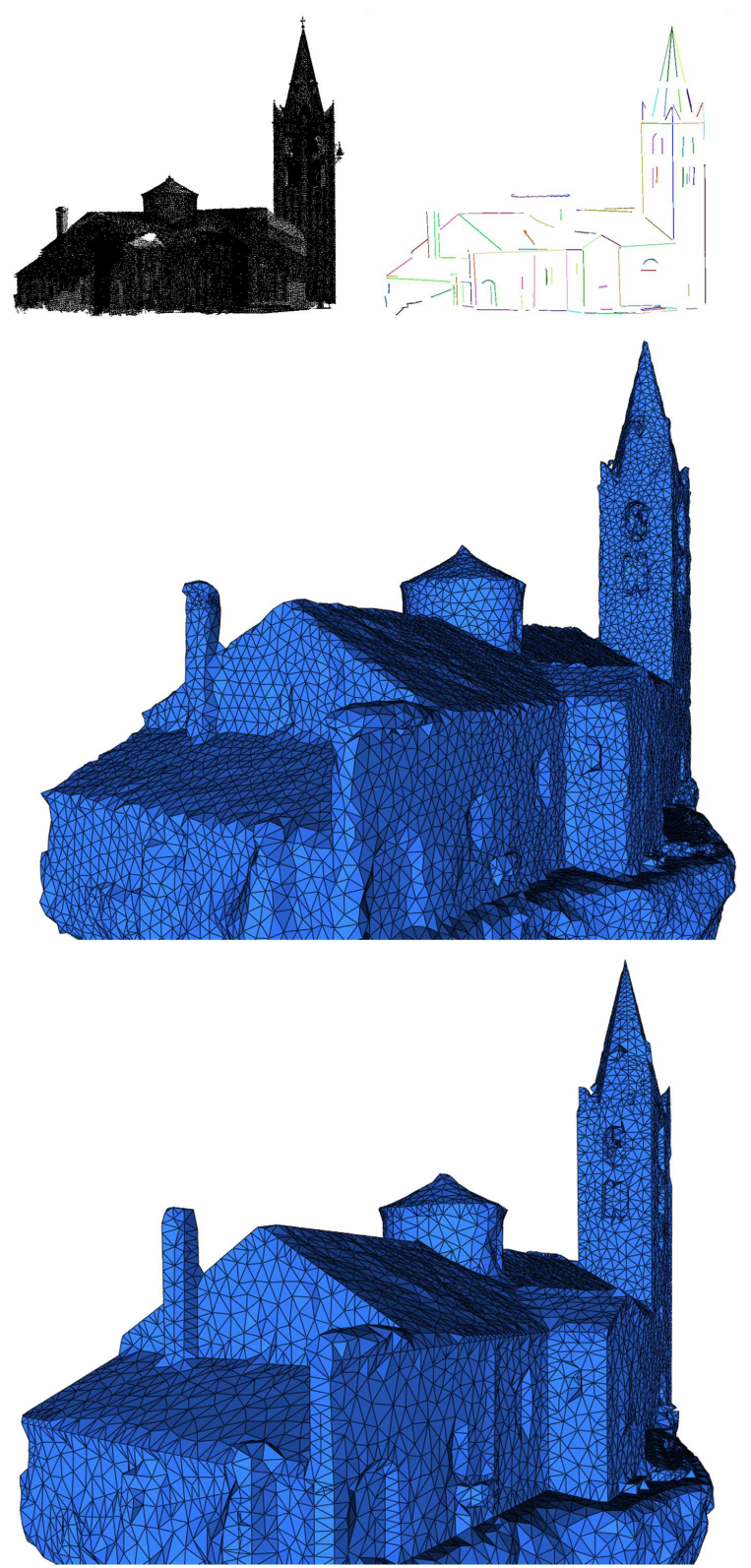

Figure 12: From top to bottom, left to right: input point cloud of Church model (courtesy of INPG) downsampled to about $7.2 \%$ of the original dataset; extracted feature polylines; meshed Poisson implicit function; output of our feature preserving mesh generation.

\section{Acknowledgements}

We wish to express our thanks to the reviewers for their insightful comments. We also thank Pierre Alliez for the precious discussions, Stephane Tayeb and Laurent Rineau to provide access to their experimental meshing code, Joel Daniels and the Aim@Shape repository for the several data-sets. This work was partially supported by the ANR (Agence Nationale de la Recherche) under the "Gyroviz" project (No ANR-07-AM-013) www.gyroviz.org and by the Fondation d'entreprise EADS, contract no 3610.

\section{References}

[AA06] Adamson A., Alexa M.: Anisotropic point set surfaces. In Proc. of Afrigraph '06 (2006), p. 13.

[AB99] AMENTA N., BERN M.: Surface reconstruction by Voronoi filtering. Discrete and Computational Geometry 22 (1999), 481-504.

[ann] ANN, a library for Approximate Nearest Neighbor searching. http://www.cs.umd.edu/ mount/ANN/.

[BO05] BoIssonnat J., OUdot S.: Provably good sampling and meshing of surfaces. Graphical Models 67, 5 (2005), 405451.

[CDL07] Cheng S., Dey T., Levine J.: A Practical Delaunay Meshing Algorithm for a Large Class of Domains*. In Proc. of IMR '07 (2007), Springer, pp. 477-494.

[CDR07] Cheng S.-W., Dey T. K., Ramos E. A.: Delaunay refinement for piecewise smooth complexes. In Proc. of SODA '07 (2007), pp. 1096-1105.

[cga] CGAL, Computational Geometry Algorithms Library. http://www.cgal.org.

[DHOS07] Daniels J. I., Ha L. K., Ochotta T., Silva C. T.: Robust smooth feature extraction from point clouds. In Proc. of SMI '07 (2007), pp. 123-136.

[DTS01] Dinh H., TURK G., Slabaugh G.: Reconstructing surfaces using anisotropic basis functions. In Proc. of ICCV 'OI (2001), pp. 606-613.

[ES97] Edelsbrunner H., SHAH N.: Triangulating Topological Spaces. International Journal of Computational Geometry \& Applications 7, 4 (1997), 365-378.

[FCOS05] Fleishman S., Cohen-Or D., Silva C.: Robust moving least-squares fitting with sharp features. In Proc. of ACM SIGGRAPH '05 (2005), p. 552.

[GG07] Guennebaud G., Gross M.: Algebraic point set surfaces. In Proc. of ACM SIGGRAPH 'O7 (2007), p. 23.

[HDD*92] Hoppe H., DeRose T., Duchamp T., McDonald J., STUETZLE W.: Surface reconstruction from unorganized points. Proc. of ACM SIGGRAPH'92 (1992), 71-71.

[JWSA08] Jenke P., WAND M., Strasser W., AKA A.: Patch-graph reconstruction for piecewise smooth surfaces. Proc. of VMV'08 (2008), 3 .

[KBH06] KaZhdan M., Bolitho M., Hoppe H.: Poisson Surface Reconstruction. In Proc. of SGP 'O6 (2006), pp. 61-70.

[KBSS01] Kobbelt L., Botsch M., Schwanecke U., SeIDEL H.: Feature sensitive surface extraction from volume data. In Proc. of ACM SIGGRAPH '01 (2001), pp. 57-66.

[LCOL07] LIPMAN Y., COHEN-OR D., LEVIN D.: Datadependent MLS for faithful surface approximation. In Proc. of SGP'07 (2007), p. 67.

[Lee00] LEE I.: Curve reconstruction from unorganized points. Computer Aided Geometric Design 17, 2 (2000), 161-177.

[MOG09] Mérigot Q., Ovsjanikov M., Guibas L.: Robust voronoi-based curvature and feature estimation. In Proc. of SIAM/ACM SPM '09 (2009), pp. 1-12.

[OBA*03] Ohtake Y., Belyaev A., Alexa M., Turk G., SEIDEL H.: Multi-level partition of unity implicits. In Proc. of ACM SIGGRAPH '03 (2003), pp. 463-470.

[OBA05] Ohtake Y., Belyaev A., Alexa M.: Sparse lowdegree implicit surfaces with applications to high quality rendering, feature extraction, and smoothing. In Proc. of SGP '05 (2005), p. 149. 
[OGG] Oztireli C., Guennebaud G., Gross M.: Feature preserving point set surfaces based on non-linear kernel regression. In Computer Graphics Forum.

[PKG03] PAuly M., KeISER R., Gross M.: Multi-scale feature extraction on point-sampled surfaces. In Computer Graphics Forum (2003), vol. 22, pp. 281-289.

[She00] SHEWCHUK J.: Mesh generation for domains with small angles. In Proc. of SCG 'OO (2000), p. 10.

[SSFS06] Schreiner J., ScheidegGer C., Fleishman S., SILVA C.: Direct (re) meshing for efficient surface processing. In Computer Graphics Forum (2006), vol. 25, pp. 527-536. 